\title{
Design optimization of non-zero dispersion shifted fiber for latency mitigation in optical fiber network
}

\begin{abstract}
Latency has an important role in new generation optical networks. There are a few ways to minimize latency in the optical networks. One way is to use silica in the core of optical fiber during the design of fabrication process. In this study, we have designed a non-zero dispersion shifted fibers (NZDSF) used as the transmission medium with minimal latency in an optical network. Using our optical fiber, the latency was improved by $0.016 \mu \mathrm{s}$.
\end{abstract}

Keywords: design optimization, low-latency, NZDSF, optical fiber network
Volume 3 Issue I - 2019

\author{
Faramarz E Seraji,' Shima Safari, ${ }^{2}$ Marzieh \\ Sadat Kiaee' \\ 'Optical Communication Group, Communication Technol Dept, \\ Iran telecom Research Center, Iran \\ ${ }^{2}$ Electrical and Computer Eng. Dept. North Branch, Islamic Azad \\ University, Iran
}

\begin{abstract}
Correspondence: Faramarz E Seraji, Faculty member Optical Communication Group, Department of Communication

Technology, Iran Telecom Research Center, Tehran, Iran, Tel +9821 8497 7723, Fax 98218863 0047, Email feseraji@itrc.ac.ir

Received: December 19, 2018 | Published: January 29, 2019
\end{abstract}

\section{Introduction}

Latency describes the time lapse between a transmitted and received signal. This parameter is crucial issue for high frequency traders associated with financial markets, In today's marketing, highfrequency trading firms pay a premium for a latency advantage as just a few microseconds of delay in receiving trading information relative to a competitor can result in loss of deals with big financial impact. ${ }^{1,2}$

Latency in a link develops as a result of the delay caused by the following different factors:

a) the signal processing in the electronics, the amplifiers, the dispersion compensating modules (if any) and

b) the delay caused by optical propagations of the signal along the fiber length, the longer the fiber, the more latency. ${ }^{1,3}$

Besides the latency contributions from each of the above factors, it is apparent that the biggest contribution comes from the transmission fibers. ${ }^{4}$ As the transmission length exceeds $10 \mathrm{~km}$, the fiber becomes almost wholly responsible for the latency. The latency introduced by the fiber, is directly proportional to the group index of the fiber. So reducing the group index can lower the latency contribution from the transmission fiber. ${ }^{4}$

Latency is one of the important issues of new generation networks. To achieve lower latency, we need to use optical fiber with lower refractive index. So the core must be doped by using silica.

A typical pure silica core ${ }^{1}$ has a $0.4 \%$ lower group index relative to traditional fibers made of Ge-doped silica core [5]. This apparent insignificant latency would be noticeable in long haul propagation across the Atlantic Ocean. This time difference will be highly attractive to traders working between stock exchanges in two respective continents.

In order to optimize the latency of an optical network at higher distances, we should optimize the parameters of the optical fiber. During the light transmission, there are several parameters that affect on the quality of the received optical signals. One of these parameters is the nonlinear effects. ${ }^{6}$ To overcome nonlinearity, large effective

${ }^{1}$ Corning's ultra-low-loss products (like SMF-28R ULL fiber and Vascade R EX2000 fiber). area fibers (LEAF) have been employed. ${ }^{7}$ In the first generation, zerodispersion shifted fibers (ZDSF) were used to achieve minimum loss and dispersion; but by increasing the number of signal wavelengths in DWDM networks, four-wave mixing (FWM) occurred which induces inter-channel crosstalk during the transmission of light. ${ }^{8,9}$

Besides ZDSFs, non-zero dispersion shifted fibers (NZDSF) have been designed which avoid the phase matching condition and reduce the effects of FWM in optical networks. ${ }^{1,4}$ By using NZDSF we may obtain a large effective area which minimizes the nonlinear effects; 7 however, larger effective area makes fiber more sensitive to bending loss. So, choosing optimum parameters during the design of an optical fiber is very crucial for optical networks performances.

To start-up, we have studied the effects of other parameters while optimizing the optical fibers. During the design process, we have faced with many limitations. Using optical fibers with very large effective area may cause a very high mode field diameter (MFD). ${ }^{10}$ In order to overcome this issue; the effective area must be limited. In a study, the upper limit of effective area in a single mode fiber was reported around $370 \mu \mathrm{m}^{2} .{ }^{11}$ In a study, an NZDSF with effective area of $95 \mu^{2}$, dispersion slop of $0.1 \mathrm{ps} / \mathrm{nm}^{2} \mathrm{~km}$ and bending loss of 0.005 $\mathrm{dB}$ has been designed. ${ }^{12}$

An NZDSF with effective area of $102 \mu \mathrm{m}^{2}$, dispersion of $4 \mathrm{ps} /$ $\mathrm{nm} . \mathrm{km}$, dispersion slope of $0.06 \mathrm{ps} / \mathrm{nm}^{2} \mathrm{~km}$ and bending loss of $0.0013 \mathrm{~dB} / \mathrm{km}$ at the wavelength $1550 \mathrm{~nm}$ was designed and reported, where one dopant was employed in the core region of the optical fiber. ${ }^{13}$

In another study, these parameters were optimized by using three dopants to make different refractive index in the core; where the effective area was increased up to $120 \mu \mathrm{m}^{2}$ and the bending loss was decreased down to $1.4 \times 10^{-14} \mathrm{~dB} / \mathrm{km}^{14}$. At wavelength $1550 \mathrm{~nm}$, the dispersion, effective nonlinear refractive index and 1'st order PMD resulted in $5.78 \mathrm{ps} / \mathrm{nm} . \mathrm{km}, 1.41 \times 10^{-16} \mathrm{~cm}^{2} / \mathrm{W}$ and $8.77 \times 10^{-2} \mathrm{ps}$, respectively.

Besides all above parameters' optimizations, one important parameter in the next generation communication systems is the signal latency between transmitting and receiving ends. As it is reported before, the typical group delay in a conventional single mode fiber is about $5 \mu \mathrm{s} / \mathrm{km} .^{15}$ 
Recently, we have designed an optical fiber network used in IoT in order to evaluate the quality of the received signals from $50 \mathrm{~km}$ of our designed NZDSF fiber. In this theoretical study, a non-zero dispersion shifted fiber (NZDSF) with a particular refractive index which had a minimum latency at $1352 \mathrm{~nm}$ was designed with an effective area of $102 \mu \mathrm{m}^{2}$ and macrobending loss of $7.31 \times 1051 \mathrm{~dB} / \mathrm{km}$. In comparison to a commercial NZDSF, the latency was improved by $0.002 \mu \mathrm{s} .{ }^{16}$

To the best of our knowledge, considerable academic works have not been reported in the literatures on the present subject. In this paper, we have attempted to optimize design of an NZDSF fiber with minimal signal latency with an optimum effective area.

\section{Refractive index profile}

As we know, the latency of light signal in a vacuum is $3.336 \mu$ s, so we must find the ways to minimize the signal latency of propagating light in an optical fiber medium for most signal velocity. To achieve this aim, we should optimize the refractive index profile of the transmission fiber for minimal group index so as to mitigate the fiber group index, thus results in higher group velocity of propagating signal. We have designed an NZDSF with a core of exponential profile assumed with Germanium dopant.

Adding Germanium or Fluorine will increase or reduce the refractive index of pure silica, respectively. The refractive index $n$ of $m$ mole-percentage doped material can be defined as follows: ${ }^{16}$

$$
n=\sqrt{n_{0}^{2}+\frac{m}{m_{1}}\left(n_{1}^{2}-n_{0}^{2}\right)}
$$

where $n_{0}$ is the refractive index of the host material and $n_{1}$ is the refractive index of doped material with $m_{1}$ mole percentage. Then the exponential function of the core is as follows : ${ }^{16}$

$$
n(x)=[n(0)-n(w)] \times \frac{e}{e-1} \times \exp (-x / w)+\frac{e . n(w)-n(0)}{e-1}
$$

where $n(0)$ and $n(w)$ are the refractive indices at $x=0$ and $x=w$, respectively, and $e=1.6 \times 10^{-19}$.

In order to calculate the latency of this fiber, we should find the group delay of light with refractive index of $n$ for a fiber length of $L$ by the first frequency-derivation of the propagation constant given as: $G_{d}=L \frac{d \beta}{d \omega}=z \frac{d \lambda}{d \omega} \frac{d\left(n k_{0}\right)}{d \lambda}=\frac{-2 \pi c L}{\omega^{2}}\left(k_{0} \frac{d n}{d \lambda}+n \frac{d n}{d \lambda}\right)=\frac{L}{c}\left(n-\lambda \frac{d n}{d \lambda}\right)$ (3)

where $N_{g}=[n-\lambda(d n / d \lambda)]$ is the effective group index of the fiber and $\lambda$ is the operating wavelength. The dispersion coefficient of the propagating light is computed by the following expression:

$$
D_{\text {total }}=-\frac{L}{c} \lambda \frac{d^{2} N_{g}}{d \lambda^{2}}
$$

As we know, more effective area may decrease the none-linearity; so it is important to minimize the nonlinearity of fiber in our design. Assume that $E(x, y)$ is the optical mode field distribution, then the effective area can be defined by using the following expression: ${ }^{16}$

$$
A_{e f f}=\frac{\left[\iint|E(x, y)|^{2} d x d y\right]^{2}}{\iint|E(x, y)|^{4} d x d y}
$$

Also, the nonlinear effective index in a fiber can be found as follows:

$$
n_{e f f}=\frac{n(x, y)|F(x, y)|^{4} d x d y}{|F(x, y)|^{2} d x d y}
$$

where $n(x, y)$ is the user-defined spatially dependent nonlinear refractive index of the various layers of the fiber and $F(x, y)$ is the normalized mode field pattern.

\section{Theory of signal latency}

The total end to end network latency is the combination of all latencies incurred between the transmitting and receiving ends and all other elements, such as witches and routers etc. Therefore, the total latency $\ell_{\mathrm{T}}$ is summarized as:

$$
\ell_{\mathrm{T}}=\ell_{\text {trans }}+\ell_{\text {rec }}+{ }_{\text {all appliancesinlink }}\left(\ell_{\text {proc }}+\ell_{\text {for }}+\ell_{\text {que }}\right)+{ }_{\text {links }} \ell_{f} \text { (7) }
$$

where $\ell_{\text {trans }}, \ell_{\text {trans }}, \ell_{\text {proc }}, \ell_{\text {for }}, \ell_{q u e}$, and $\ell_{f}$ are latencies due to transmitter, receiver, processing, forwarding, queuing, and propagation of optical signals in the fiber, respectively

To focus our study, we consider the latency due to signal propagation in the transmission fiber which causes a major latency in an optical fiber network. The speed of an optical signal in a fiber is known as group velocity $v_{g}$ that can be determined by the effective group refractive index $N_{g}$ by:

$$
v_{g}=\frac{c}{N_{g}}
$$

where $c$ is the velocity of light in a vacuum. By knowing the length of the transmission fiber $L$ and group velocity, the fiber latency can simply be calculated as:

$$
\mathbf{I}_{f}=\frac{L}{v_{g}}=\frac{L N_{g}}{c}
$$

\section{Design procedure}

We have started our study by using the conventional NZDSF of Corning Inc. with dispersion of $4 \mathrm{ps} / \mathrm{nm}$. km, effective area of $72 \mu \mathrm{m}^{2}$ and effective group refractive index of 1.4693. Therefore, the latency $\mathbf{I}_{1550}$ of this optical fiber at wavelength $1550 \mathrm{~nm}$ for the given values is calculated as follows:

$$
\begin{aligned}
& V_{1550 \mathrm{~nm}}=c / N_{g}=\frac{299792.458}{1.4693}=204037.608 \mathrm{~km} / \mathrm{s} \\
& \mathbf{I}_{1550}=\frac{1_{\mathrm{km}}}{V_{1550 \mathrm{~nm}}}=\frac{1}{204037.608}=4.901 \mu \mathrm{s}
\end{aligned}
$$

where $V$ is the velocity of light in fiber, $c$ is the velocity of light in a vacuum, $N_{g}$ is the effective group refractive index.

We have started our design procedure by employing the profile of an optical fiber which was used to achieve high negative dispersion in a dispersion compensating fiber (DCF). ${ }^{17}$ Since our first aim was to design an NZDSF with an appropriate dispersion, we have modified the sample core profile into an exponential function.

There are some literature reports that revealed the exponential profile would decrease the loss level..$^{18,19}$ Accordingly, we have used two silica-based dopants with refractive index of 1.4-1.46 in the cladding region. By employing the proposed parameters' values of the refractive profile given in Table 1, we have proposed a profile as illustrated in Figure 1, showing the refractive index and percentage of the refractive index difference.

The refractive index profile is divided into 5 Regions. The Region 1 forms the core of the fiber with a radius of $2.2 \mu \mathrm{m}$ with an exponential variation of the refractive index changing from 1.446 to 1.460 . The Region 2 is a gap of $0.5 \mu \mathrm{m}$ with refractive index of 1.4448 between 
the core and the Region 3 with a width of $0.8 \mu \mathrm{m}$ and refractive index of 1.45 , considered as a refractive index ring at the vicinity of the core in the cladding.

Table I The proposed parameters' values of the refractive index profile

\begin{tabular}{|c|c|c|c|c|c|}
\hline \multirow{2}{*}{ Parameters } & \multicolumn{5}{|l|}{ Regions } \\
\hline & \# 1 (Core) & $\# 2$ & $\# 3$ & $\# 4$ & $\# 5$ \\
\hline Profile regions & Exponential & Gap & Ring & Gap & Clad \\
\hline Width ( $\mu \mathrm{m})$ & 2.2 & 0.5 & 0.8 & 1 & 58 \\
\hline Refractive Index & $\begin{array}{l}1.460 \text { Max- } \\
1.446 \text { Min }\end{array}$ & 1.4448 & 1.45 & 1.4448 & 1.4462 \\
\hline
\end{tabular}

Region 4 is yet another gap between the ring and the cladding with a width of $1 \mu \mathrm{m}$ and refractive index of 1.4448 . The final Region 5 is forming the major part of the cladding with a span of 58 and refractive index of 1.4462, as shown in Figure 1.

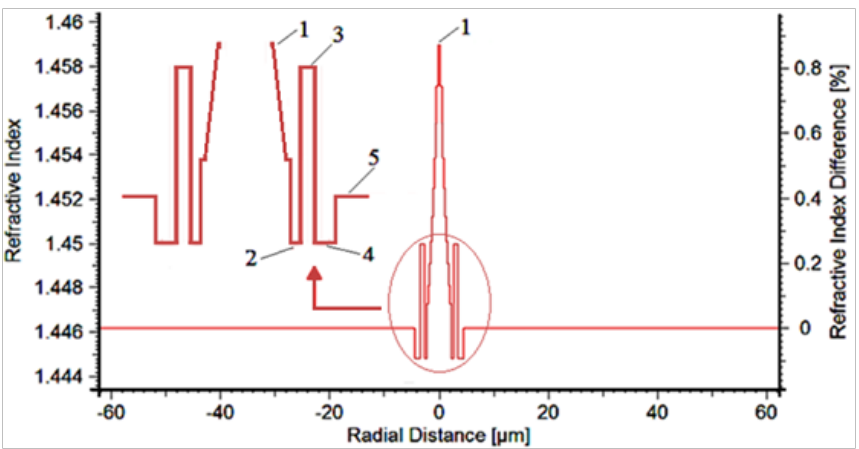

Figure I Exponential refractive index profile of the designed NZDSF.

Figure 2 shows the dispersion of the designed profile. As it is noted, total dispersion and dispersion slope are $3.089 \mathrm{ps} / \mathrm{nm} . \mathrm{km}$ and $0.0973 \mathrm{ps} / \mathrm{nm}^{2} . \mathrm{km}$, respectively, at wavelength $1550 \mathrm{~nm}$. The effective area of our profile is $130 \mu \mathrm{m}^{2}$. Because of this large effective area, there will be a limitation in bending, so also in the bending loss, as depicted in Figure 3. Above $1550 \mathrm{~nm}$, the macro-bending loss increases with a higher slope.

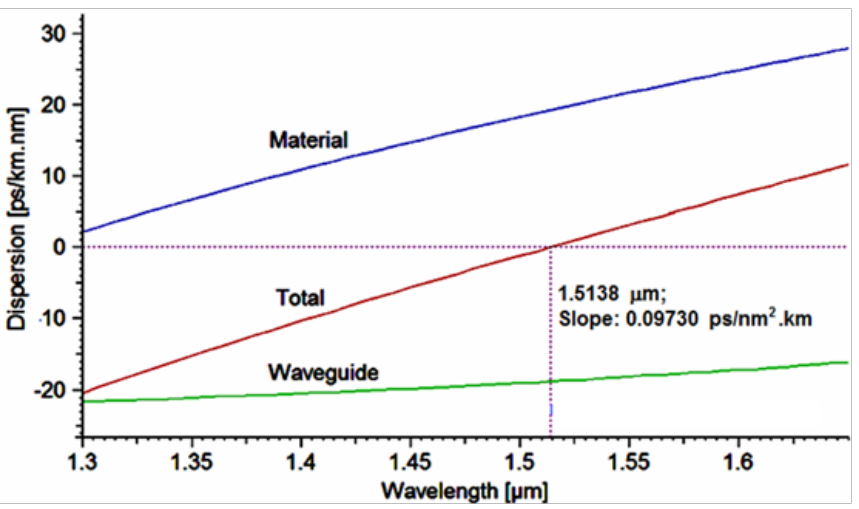

Figure 2 Dispersion of the designed NZDSF profile versus wavelength I.5। $38 \mu \mathrm{m}$, Slope: $0.09730 \mathrm{ps} / \mathrm{nm} 2 . \mathrm{km}$

The macro- and micro-bending losses in this profile are 0.79 and $1.28 \mathrm{~dB} / \mathrm{km}$, respectively. Material loss is $0.19 \mathrm{~dB} / \mathrm{km}$ and splices loss in the condition of splicing two matched optical fibers, is $4.77 \mathrm{~dB}$.

We have found that the minimum latency is obtained at the wavelength of $1510 \mathrm{~nm}$ which is $1.4884 \mu \mathrm{s}$. Using Eq. 3 the group index of the above profile is 1.4644 at the wavelength $1550 \mathrm{~nm}$.
Replacing this amount in Eqs. 8 and 9, the estimated latency is obtained as $4.31 \mu \mathrm{s}$. But the latency at the same wavelength is $4.884 \mu \mathrm{s}$; so we have $0.574 \mu$ s tolerance between the estimated and practical latency.

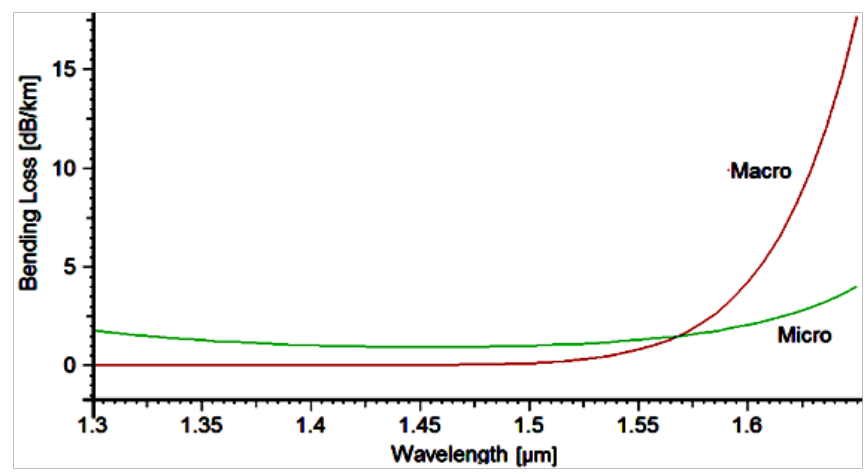

Figure 3 Bending losses of the designed NZDSF profile versus wavelength.

In Table 2, our obtained results are tabulated and are compared to the data of the large effective area fibers reported in Coning data sheet.

Table 2 Designed values compared to the data of NZDSF reported by Coning Inc

\begin{tabular}{|c|c|c|c|c|}
\hline \multirow{2}{*}{ Parameters } & \multicolumn{2}{|c|}{ Our model @ } & \multicolumn{2}{|c|}{ Corning's NZDSF @ } \\
\hline & $1550 \mathrm{~nm}$ & $1310 \mathrm{~nm}$ & $1550 \mathrm{~nm}$ & $1310 \mathrm{~nm}$ \\
\hline Group index & 1.4644 & 1.4648 & 1.4693 & - \\
\hline $\begin{array}{l}\text { Group delay } \\
(\mu \mathrm{s} / \mathrm{km})\end{array}$ & 4.885 & 4.886 & 4.901 & - \\
\hline $\begin{array}{l}\text { Group velocity } \\
(\mathrm{km} / \mathrm{s})\end{array}$ & 204720.335 & 204664.431 & 204037.608 & - \\
\hline $\begin{array}{l}\text { Latency } \\
(\mu \mathrm{s} / \mathrm{km})\end{array}$ & 4.885 & 4.886 & 4.901 & \\
\hline $\begin{array}{l}\text { Dispersion (ps/ } \\
\mathrm{nm} . \mathrm{km})\end{array}$ & 3.23 & -21.59 & 6-Apr & - \\
\hline $\begin{array}{l}\text { Effective area } \\
\left(\mu \mathrm{m}^{2}\right)\end{array}$ & 130 & 60 & 72 & - \\
\hline $\begin{array}{l}\text { Material loss } \\
(\mathrm{dB} / \mathrm{km})\end{array}$ & 0.19 & 0.36 & 0.19 & $<0.4$ \\
\hline $\begin{array}{l}\text { Macrobending } \\
\text { loss }(\mathrm{dB} / \mathrm{km})\end{array}$ & 0.81 & 1.63 & $<0.5$ & - \\
\hline PMD (ps) & 18.06 & - & 9.5 & - \\
\hline
\end{tabular}

For the next step, we have employed our designed NZDSF in an optical network to evaluate how far the light will be transmitted without using EDFA and DCF and can be detected with desirable quality. For this aim, we have designed an optical network based on an optical transmitter and a receiver with the bit rate of $10 \mathrm{~Gb} / \mathrm{s}$. The received signal at the distance of $80 \mathrm{~km}$ was monitored by using eye-diagram as shown in Figure 4 . The quality factor and minimum bit-error rate of this signal were 10.91 and $5.03 \times 10^{-28}$, respectively, which are desirable amounts for this distance of transmission. ${ }^{20,21}$

\section{Conclusion}

In this study, we have designed an NZDSF with refractive index of 1.4644 at wavelength of $1550 \mathrm{~nm}$ which had minimum latency at 1352 $\mathrm{nm}$. In comparison to Corning NZDSF, the latency for the designed NZDSF has improved about $0.016 \mu$ s per kilometer The effective area is $130 \mu \mathrm{m}^{2}$ and macro-bending loss is obtained as $0.79 \mathrm{~dB} / \mathrm{km}$. 
The dispersion of this fiber is $3.23 \mathrm{ps} / \mathrm{nm} . \mathrm{km}$ which is comparable to Corning NZDSF fiber.

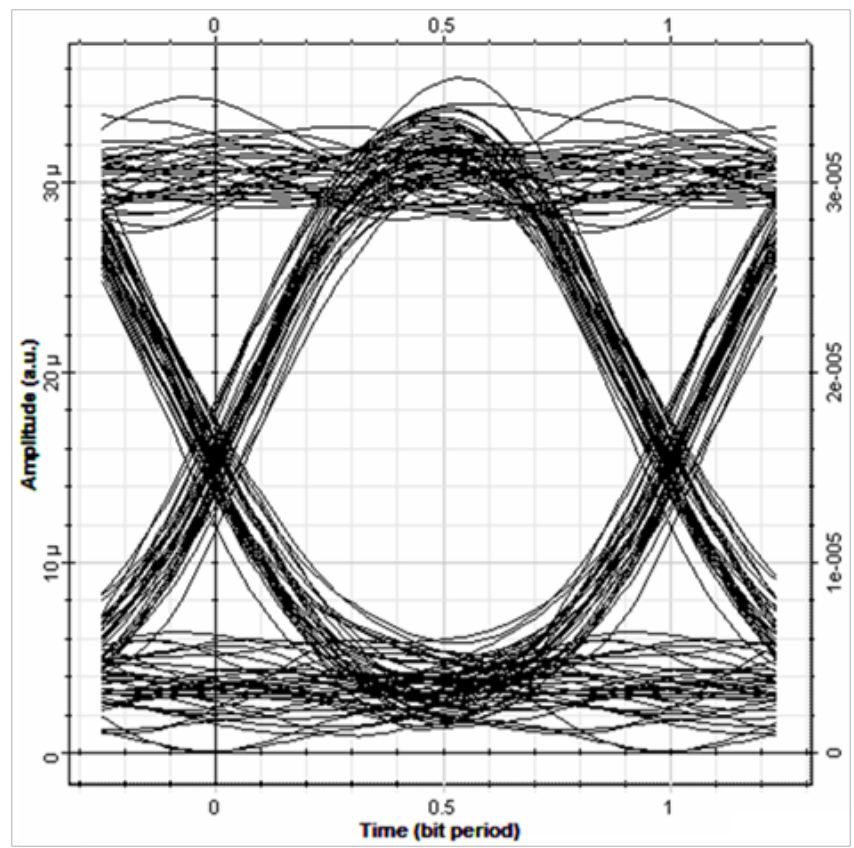

Figure 4 Eye-diagram of the received signals at the distance of $80 \mathrm{~km}$.

We have employed our designed NZDSF in an optical fiber network in order to evaluate the quality of the received signals from $80 \mathrm{~km}$ of our fiber. The results have revealed that without using optical amplifier and DCF, the quality factor and minimum bit-error rate have been obtained as 10.91 and $5.03 \times 10^{-28}$ respectively.

\section{Acknowledgments}

The authors acknowledge the allocated study mission project with the Permit No. 2210, in Communication Technology Dept., at Iran Telecom Research Center.

\section{Conflicts of interest}

Authors declare there is no conflicts of interest.

\section{References}

1. Narottam Das. Advances in Optical Communication. UK: Intechopen Limited; 2014.

2. Low-Latency Market Data. Ireland: Corvil Insight Inc; 2010.

3. Kawanishi T, Kanno A, Yoshida Y, et al. Impact of wave propagation delay on latency in optical communication systems. Proceedings Volume 8646, Optical Metro Networks and Short-Haul Systems V. USA; 2013.

4. Mazzarese D. Minimizing latency in long-haul networks. Lightwave. 2011.
5. Corning Inc. Corning SMF-28e+optical fiber specification datasheet. 2013.

6. Kazumasa A, Ohsono, Tomoyuki Nishio, et al. Low non-linear nonzero dispersion-shifted fiber for DWDM transmission. Hitachi Cable Review. 2000;19.

7. Arai S, Akasaka Y, Suzuki Y, et al. Low nonlinear dispersion-shifted fiber. OFC'97 Technical Digest. 1997; 65 p.

8. Wandel M, Kristensen P. Fiber designs for high figure of merit and high slope dispersion compensating fibers. J Opt Fiber Commun Res. 2006;3(1):25-60.

9. Paula B Harboe, Edilson da Silva, José R Souza. Analysis of FWM penalties in DWDM systems based on G.652,G.653, and G.655 optical fibers. World Academy of Science, Engineering and Technology. 2008;48(12):77-83.

10. Kant S, Sahu H, Arora A. Enhanced bend insensitive high effective area NZDS fiber. International Wire and Cable Symposium, Proceedings of the 55th IWCS/Focus. 2010. p. 330-333.

11. Li MG, Chen X, Liu A, et al. Limit of Effective Area for Single-Mode Operation in Step-Index Large Mode Area Laser Fibers. Journal of Lightwave Technology. 2009;27(15):3010-3015.

12. Siliard P, Molin D. Non-zero dispersion shifted optical fiber having a large effective area. Patent application number: 20110188826; 2011.

13. Shizhuo Yin, Kun-Wook Chung, Hongyu Liu, et al. A new design for NZDSF with a large effective area over $100\left(\mu^{2}\right)$ and low bending and splice loss. Optics Communications. 2000;177(1-6):225-232.

14. Sivanantharaj Avaninathan, S Selvedran, et al. An optimized design for NZDSF with reduced nonlinear effects for future optical networks. J Optica Applicata. 2014;44(4):503-519.

15. John A Jay. Low signal latency in optical fiber networks. International Wire \& Cable Symposium. Proceedings of the 60th IWCS Conference. 2011. p. 429-437.

16. Seraji FE, Kiaee MS. Design optimization of NZDSF for low latency in iot optical fiber network. Phys Astron Int J. 2018;2(5):448-450.

17. Faramarz E. Seraji, Razieh Kiaee. A Revisit of Refractive Index Profiles Design forReduction of Positive Dispersion, Splice Loss, and Enhancement of Negative Dispersion in OpticalTransmission Lines. Int J Opt Apps. 2014;4(2):62-67.

18. Marc Nikles, Luc Thevenaz, Philippe A Robert. Brillouin Gain Spectrum Characterization in SMF. J Lightwave Technol. 1997;15(10):18421851.

19. KZ Nobrega, ASB Sombra. Optimum self-phase modulation profile for nonlinear transmission recovery in twin core optical couplers with loss. J Opt Commun. 1998;151:1-3.

20. Go Yun Li, K Dimyati. Evaluation of Direct Modulation and External Modulation in Temporal Phase Encoding System. IEEE Malaysia Int Conf; 2013 Nov 26-28; IEEE. 2013. p. 425-455.

21. Muthana Y Aldouri, SA Aljunid, R Badlishah Ahmad, et al. BER Performance of RZ and NRZ Data Signals Optical Code Division Multiple Access System Based on and Detection Scheme in Fiber to the Home Network. Optica Apllicata. 2011;21(10): 9-13. 